$10.1117 / 2.1200608 .0274$

\title{
Optical microscopy on the Internet
}

\section{Michael Davidson}

Florida State University is adapting educational websites it has built to cover the increasing application of optical microscopy in the biological and materials sciences.

Although the Internet provides easy, comprehensive access to scientific literature about research that uses optical microscopy, it falls short in other respects. For example, the development of educational websites targeting beginning students and novices in the field has lagged far behind. Also, it doesn't adequately catalogue the online resources of many relevant commercial aftermarket instrument manufacturers, such as those offering automatic microscope accessories and live-cell imaging chambers.

Useful website content that could remedy this situation would include equipment and specimen information, image galleries, and tutorials. Virtually every laboratory involved with confocal microscopy-which uses imaging techniques that increase contrast and allow 3D specimen reconstructioncould maintain such sites, but few do. Several sites have been developed by researchers ${ }^{1}$, students, ${ }^{1}$ and microscope manufacturers ${ }^{2}$ on current topics related to confocal microscopy, but these are limited in scope. Exhaustive Web searches have revealed scant educational information other than hyperlinks at PubMed to original research articles that are often difficult for beginning students to understand.

Florida State University (FSU) is addressing these shortcomings by bringing the resources of several educational websites to bear on the growing application of optical microscopy in biological and materials sciences. This effort augments existing sites with content that focuses on important educational issues in microscopy, and provides links to confocal scientific literature and the websites of manufacturers who supply useful accessories. The new content includes well-illustrated review articles, interactive Java tutorials, image galleries, and links to original research reports at article-indexing services.

One of the FSU websites, called Molecular Expressions, ${ }^{3}$ was developed by the National High Magnetic Field Labora- tory's Optical Microscopy Research Group, which has a decade of experience providing online resources for undergraduate and graduate students. With over 9,500 HTML pages and 75 image galleries, Molecular Expressions is the group's largest such endeavor. Based on distinct IP addresses, the site hosts about 30,000 unique daily visitors. Its image galleries feature a variety of contrast-enhancing techniques, such as fluorescence, darkfield, differential interference contrast, confocal, Hoffman modulation contrast, brightfield, and phase contrast. The site also offers hundreds of review articles on optical microscopy, ${ }^{4}$ and over 500 interactive Java tutorials that allow the visitor to adjust variables in a manner similar to operating an actual microscope.

Topics covered at Molecular Expressions include an introduction to the physics of light and color, basic principles of the optical microscope, advanced and specialized contrast-enhancing techniques, fluorescence, photomicrography, digital image acquisition and processing, stereomicroscopy, laser scanning confocal microscopy, and a 'virtual' museum of microscopes developed through the ages. ${ }^{5}$

Similar websites built and maintained by FSU are offered as a courtesy to the general public by Nikon (Microscopy $\mathrm{U}^{6}$ ) and Olympus (Microscopy Resource Center ${ }^{7}$ ). Recently, Olympus introduced another site dealing specifically with confocal microscopy (Olympus FluoView Resource Center ${ }^{8}$ ).

Figure 1 illustrates two typical examples of material presented as Java-based tutorials designed for beginning students. The tutorial shown in Figure 1(a) demonstrates the fundamentals of the optical astigmatism aberration, which students learn is manifested by the off-axis image of a specimen point appearing instead as a line or ellipse.

Somewhat more complex in nature, the tutorial illustrated in Figure 1(b) demonstrates the principles of a de Sénarmont compensator, which is implemented in several commercial differential interference-contrast microscopes. The compensator combines a linear polarizer with a quarterwavelength retardation plate to produce linear, elliptical, or 

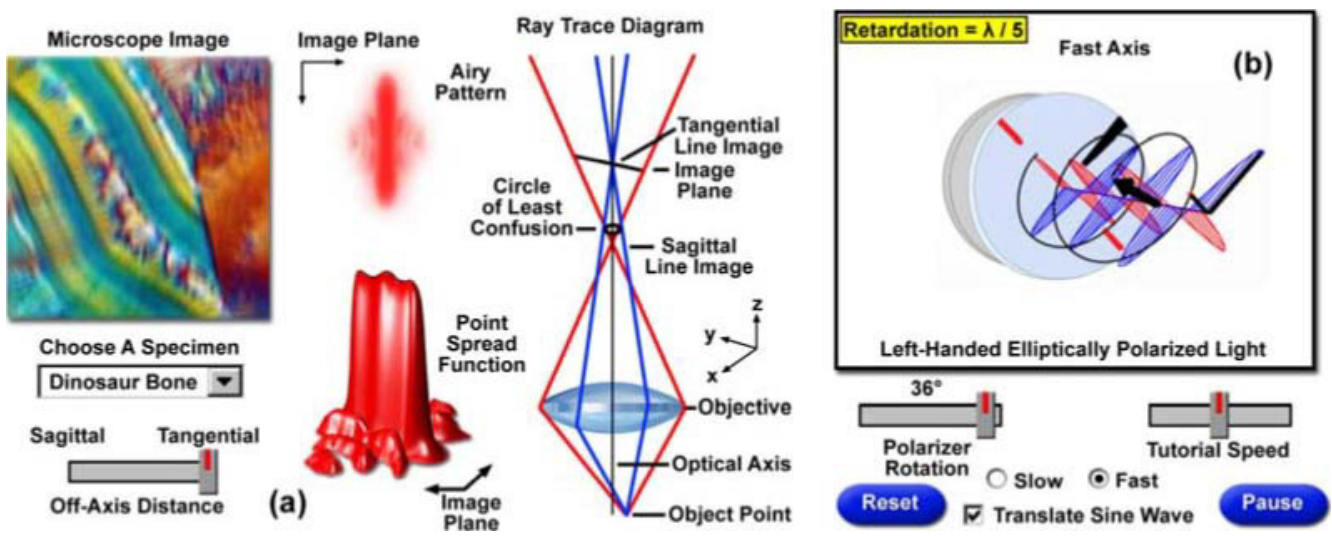

Figure 1. Shown are interactive Java tutorials demonstrating (a) astigmatism, a common optical aberration, and (b) the production of elliptically polarized light by a de Sénarmont compensator. The tutorials are controlled with sliders, pull-down menus, and radio buttons. Changes to the Airy pattern, point spread function, specimen image, and ray trace diagram in (a) are continuously updated as the slider position is altered. In (b), the wavefront trajectories are dynamically controlled by the sliders, and the entire compensator can be rotated using the mouse cursor.

circularly polarized light, depending on the orientation of the polarizer vibration axis with respect to the retardation plate's fast and slow axes. Students can interactively explore the relationship between wavefronts emanating from the compensator as the polarizer is rotated through its useful range.

The newest portions of these websites are devoted to the rapidly evolving field of fluorescence microscopy, which is the current focus of the development team's effort. As with the other subjects in FSU's educational initiative, educational tutorials and review articles will be used to highlight the subject, which includes such topics as spectral imaging, spinning disk and swept field live motion confocal techniques, laser scanning confocal, TIRF (total internal reflection fluorescence), FRAP (fluorescence recovery after photobleaching), FRET (fluorescence resonance energy transfer), and photoactivation.

\section{Author Information}

\section{Michael Davidson}

National High Magnetic Field Laboratory

Florida State University

Tallahassee, FL

\section{References}

1. J. P. Robinson, Purdue University Cytometry Laboratories, Purdue University, West Lafayette, Indiana, 2006. http:/ / www.cyto.purdue.edu/

2. Webmaster et al., PerkinElmer Live Cell Solutions, PerkinElmer, Boston, Massachusetts, 2006.

http:/ /las.perkinelmer.com/content/livecellimaging/solutions.asp

3. M. W. Davidson et al., Molecular Expressions, National High Magnetic Field Laboratory, Florida State University, Tallahassee, 2006. http:/ / www.microscopy.fsu.edu /
4. M. Abramowitz, E. Keller, K. R. Spring, D. B. Murphy, M. J. ParryHill, J. Swedlow, J. Lippincott-Schwartz, G. Patterson, D. W. Piston, and M. W. Davidson, The Molecular Expressions Microscopy Primer, National High Magnetic Field Laboratory, Florida State University, Tallahassee, 2006. http:/ / www.microscopy.fsu.edu/primer/

5. M. W. Davidson et al., The Molecular Expressions Museum of Microscopy, National High Magnetic Field Laboratory, Florida State University, Tallahassee, 2002. http:/ / www.microscopy.fsu.edu/primer/museum/

6. S. Schwartz, S. Ross, K. R. Spring, T. J. Fellers, M. J. Parry-Hill, D. B. Murphy, J. Lippincott-Schwartz, G. Patterson, D. W. Piston, and M. W. Davidson, Nikon Microscopy U, National High Magnetic Field Laboratory, Florida State University, Tallahassee, 2006. Nikon MicroscopyU, http:/ / www.microscopyu.com/

7. M. Abramowitz, W. K. Fester, T. J. Fellers, K. R. Spring, M. J. Parry-Hill, D. B. Murphy, and M. W. Davidson, The Olympus Microscopy Resource Center, National High Magnetic Field Laboratory, Florida State University, Tallahassee, 2006. http:/ / www.olympusmicro.com/

8. M. W. Davidson et al., Olympus FluoView Resource Center, National High Magnetic Field Laboratory, Florida State University, Tallahassee, 2006. http:/ / www.olympusconfocal.com/ 